\title{
Geovisualization of complex origin-destination flow maps using Discrete Global Grid Systems
}

\author{
Paulo Raposo $^{\text {a }}$ \\ ${ }^{a}$ Department of Geography, The University of Tennessee, Knoxville, USA, praposo@utk.edu
}

Keywords: OD maps, flow maps, DGGS, geovisualization

\begin{abstract}
:
Flow maps, where counts of people or things are shown travelling between origin-destination pairs with paths or arrows, are difficult to draw well, mostly because they rapidly get cluttered as more and more paths are added. Researchers in cartographic and information visualization fields have sought to mitigate these problems in various ways, including cluster detection and edge bundling (Buchin, Speckmann, \& Verbeek, 2011; Guo, 2009), force-directed path placement (Holten \& van Wijk, 2009; Jenny et al., 2017), use of network topological space instead of planimetric space (Xiao \& Chun, 2009), interactive spatial filtering of flows (Vrotsou, Fuchs, Andrienko, \& Andrienko, 2017), and by changing the drawing medium to accommodate flows that arc through 3D space (Raposo, 2017; Yang et al., 2019; Zhang, Zhang, Li, \& Li, 2018). Other geovisualization techniques recently developed involve and use of matrixes or grids to store and visualize flows; Wood, Dykes, \& Slingsby (2010) use spatial tessellation and a set of small multiples of the same tessellation representing origin and destination spaces, respectively, creating a sort of travel heat map when tiles are colored as a choropleth. A comparable approach is taken by Yang et al. (2017) in a system that combines an abstract matrix of flow records with inflow and outflow maps.
\end{abstract}

This early-stage project builds on previous matrix-based flow visualization methods while leveraging the power of Discrete Global Grid Systems (DGGS), which present a naturally Earth-oriented hierarchical tessellation upon which a matrix of origins and destinations can be stored and visualized. DGGS (Sahr, White, \& Kimerling, 2003; Raposo, Robinson, \& Brown, in press) partition the round Earth into nested tiles in a manner similar to quad-trees, and have the desirable properties of covering the whole spherical globe in equal-area (or near equal-area) tiles, and of being hierarchical to an arbitrary number of levels. The hierarchical quality allows flows in a DGGS to be naturally bundled by origin and destination points as a function of grid size; greater or lesser detail (i.e., more or less bundling) in the overall pattern of flows is effected by descending or ascending DGGS levels. As DGGS are becoming increasingly popular for geospatial data computation and collection in movement analysis (Brodsky, 2018), the present work provides a natural bridge to the visualization of such data when it pertains to movement phenomena.

The present research will develop a suite of flow visualization techniques upon an existing open-source DGGS rendering platform (Raposo, Robinson, \& Brown, in press): 1) grid facet coloring in a choropleth scheme to indicate magnitudes of flow into or out of a particular facet; 2) vertical-space cubic spline flow arcs, and 3) tabular representation of the flow data. All three visualization techniques will function across multiple spatial resolutions, corresponding to each of the hierarchical levels of our chosen DGGS, so that users can interact both with each of the three visualizations of the data as well as the spatial granularity to which the data are displayed. All views will be interactive and linked.

We use Dutton's (1999) quaternary triangular mesh (QTM), being a DGGS based on recursively subdividing the faces of an octahedron over the globe (Figure 1). Our method begins by finding, at many nested QTM levels, the facets of the QTM with which each origin and destination point intersects; in practice, we use up to 16 levels, as the triangles at that level in the QTM are small enough to address typical individual buildings. Importantly, point-in-polygon intersection is calculated geodetically, and not in 2D projected space, since failing to do this can cause topological errors (Raposo, Robinson, Brown, in press). At each QTM facet and at each level, we build two lists: one of all the other facets at that level that contain an origin point for a flow that arrives at this facet, and another of those that contain a destination point for flows originating in this facet. These lists are stored to each facet as polygon attributes. A flow path curving through vertical space via a cubic spline is also derived for each origin-destination pair and stored as a renderable 3D solid.

The QTM is then plotted in our software on a virtual globe using NASA's World Wind application programming interface. As users select any one QTM facet, its attributes are read to find all the other facets either contributing or receiving flows to or from the selected facet; these are then colored according to a choropleth scheme to visualize magnitudes of flow (Figure 2). Coloration is controlled for various descriptive statistics on the flows (e.g., total, mean, maximum, etc.). Linear paths curving up and around the globe (Figure 3) will also be available to be toggled on or off, giving users multiple redundant symbolizations of travel paths. 


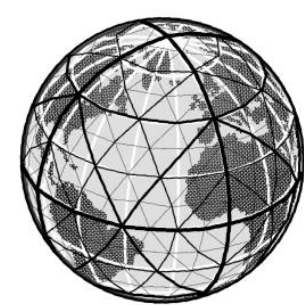

Figure 1. Dutton's (1999) QTM discrete global grid system, to three levels of detail.

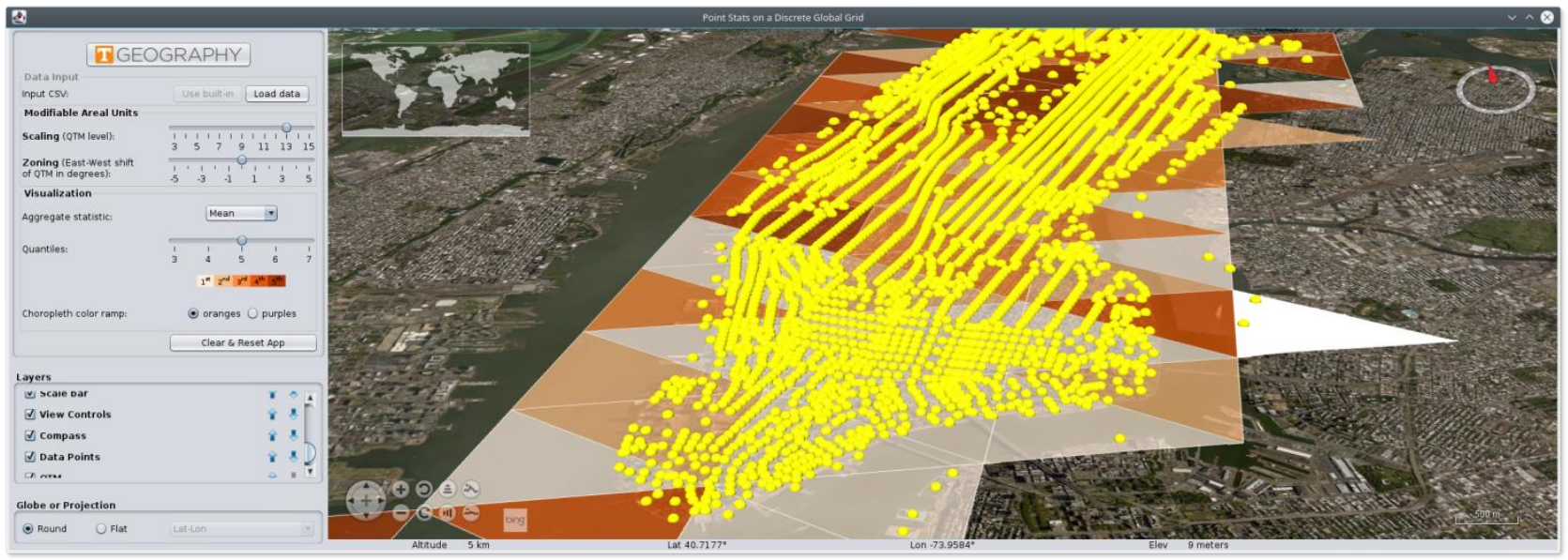

Figure 2. An illustration of existing research software, demonstrating the choropleth portion of how flow visualization will be implemented, over Manhattan. Points of origin and destination are aggregated to the facets of a DGGS, and when one facet is highlighted (at right in white in the image above), the others take on colors representing the number of flows out of or into themselves, to or from the highlighted facet. The scene changes interactively.

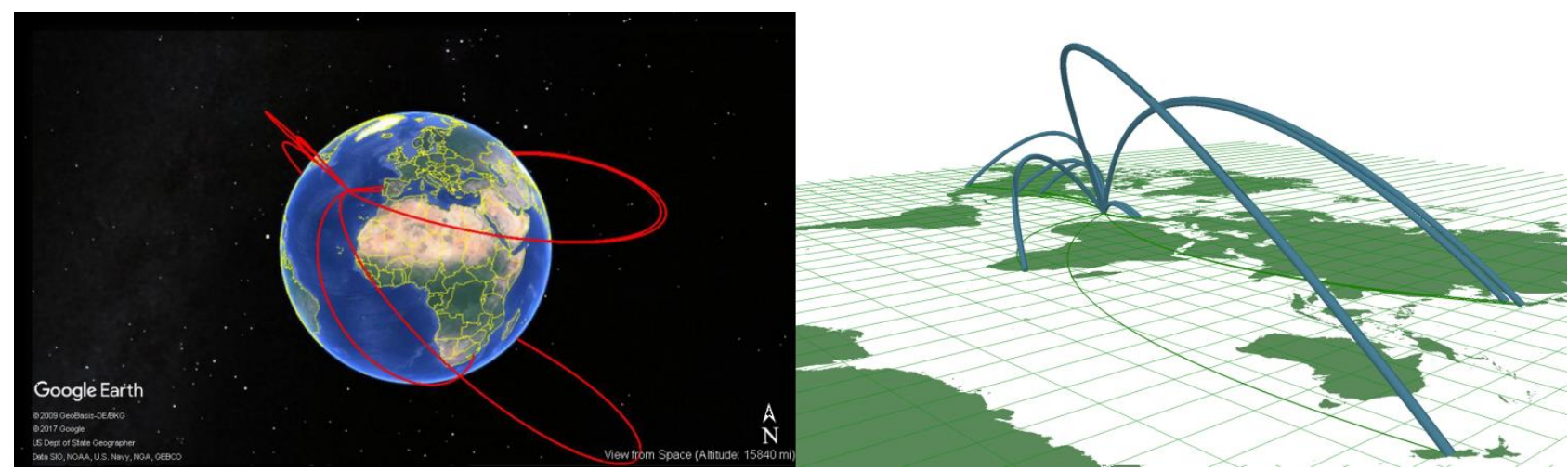

Figure 3. Prototypes of 3D flow paths developed, to be integrated into the DGGS application.

\section{References}

Brodsky, I. (2018, June). h3: Hexagonal hierarchical geospatial indexing system. Uber Open Source. Retrieved 2018 06-29, from https://github.com/uber/h3

Buchin, K., Speckmann, B., \& Verbeek, K. (2011). Flow Map Layout via Spiral Trees. IEEE Transactions on Visualization and Computer Graphics, 17(12), 2536-2544. https://doi.org/10.1109/TVCG.2011.202

Dutton, G. H. (1999). A Hierarchical Coordinate System for Geoprocessing and Cartography (1st ed.). Berlin, Heidelberg: Springer-Verlag. Retrieved from https:/www.springer.com/us/book/9783540649809

Holten, D., \& van Wijk, J. J. (2009). Force-Directed Edge Bundling for Graph Visualization. Computer Graphics Forum, 28(3), 983-990. https://doi.org/10.1111/j.1467-8659.2009.01450.x

Jenny, B., Stephen, D. M., Muehlenhaus, I., Marston, B. E., Sharma, R., Zhang, E., \& Jenny, H. (2017). Force-directed layout of origin-destination flow maps. International Journal of Geographical Information Science, 31(8), 1521-1540. https://doi.org/10.1080/13658816.2017.1307378 
Raposo, P. (2017). Open-Source Flow Maps with Cubic Splines. Presented at the North American Cartographic Information Society (NACIS) Annual Meeting, Montreal, Canada.

Raposo, P., Robinson, A., \& Brown, R. (in press). A Virtual Globe Using a Discrete Global Grid System to Illustrate the Modifiable Areal Unit Problem. Cartographica.

Sahr, K., White, D., \& Kimerling, A. J. (2003). Geodesic Discrete Global Grid Systems. Cartography and Geographic Information Science, 30(2), 121-134. https://doi.org/10.1559/152304003100011090

Vrotsou, K., Fuchs, G., Andrienko, N., \& Andrienko, G. (2017). An Interactive Approach for Exploration of Flows Through Direction-Based Filtering. Journal of Geovisualization and Spatial Analysis, 1(1), 1. https://doi.org/10.1007/s41651-017-0001-7

Wood, J., Dykes, J., \& Slingsby, A. (2010). Visualisation of Origins, Destinations and Flows with OD Maps. The Cartographic Journal, 47(2), 117-129. https://doi.org/10.1179/000870410X12658023467367

Xiao, N., \& Chun, Y. (2009). Visualizing Migration Flows Using Kriskograms. Cartography and Geographic Information Science, 36(2), 183-191. https://doi.org/10.1559/152304009788188763

Yang, Y., Dwyer, T., Goodwin, S., \& Marriott, K. (2017). Many-to-Many Geographically-Embedded Flow Visualisation: An Evaluation. IEEE Transactions on Visualization and Computer Graphics, 23(1), 411-420. https://doi.org/10.1109/TVCG.2016.2598885

Yang, Y., Dwyer, T., Jenny, B., Marriott, K., Cordeil, M., \& Chen, H. (2019). Origin-Destination Flow Maps in Immersive Environments. IEEE Transactions on Visualization and Computer Graphics, 25(1), 693-703. https://doi.org/10.1109/TVCG.2018.2865192

Zhang, M.-J., Zhang, K., Li, J., \& Li, Y.-N. (2018). Visual Exploration of 3D Geospatial Networks in a Virtual Reality Environment. The Computer Journal, 61(3), 447-458. https://doi.org/10.1093/comjn1/bxx117 